ISSN: 0258-9702 ISSN L: 2710-7647

Volumen: 30 Fecha de recibido: 16/10/2019 Fecha de publicación: Enero-Junio de 2020

Año: 2020 Fecha de aceptado: 19/11/2019 Correo: revista.sciencia@up.ac.pa

Numero: 1 Número de páginas: 53-59 URL:https://revistas.up.ac.pa/index.php/scientia

\title{
Evaluación de la eficiencia de diferentes tipos de trampas de pseudotallo, para la captura del picudo negro del plátano (Cosmopolites sordidus, Germar, 1824), en la provincia de Darién, república de Panamá.
}

\section{EDDY BARRAZA A. ${ }^{1}$, SILVIA CHAVARRÍA. ${ }^{2}$}

${ }^{1}$ Universidad de Panamá, Facultad de Ciencias Agropecuarias, Departamento de Protección Vegetal, Panamá, Panamá eddyup.pa@gmail.com

${ }^{2}$ Instituto de Investigaciones Agropecuarias de Panamá, IDIAP, Panamá, Panamá chavarriasilvia14@gmail.com

\section{RESUMEN}

Se realizó un estudio para evaluar la eficiencia de diferentes tipos de trampas de pseudotallo en la captura de insectos adultos de Cosmopolites sordidus, en parcela comercial de plátano en la provincia de Darién. Los tipos de trampas evaluados fueron; sándwich cilíndrica, tipo bisel, cepa en pie tipo V y pitfall; determinando la cantidad de individuos capturados por trampa. Las muestras recolectadas fueron analizadas en el Laboratorio de Protección Vegetal de la Facultad de Ciencias Agropecuarias de la Universidad de Panamá. El diseño experimental fue de bloques completamente al azar, con cuatro tratamientos y cuatro repeticiones. Los resultados obtenidos fueron sometidos al análisis de varianza y test de DMSt, al 5\% de probabilidad. Los mejores resultados se obtuvieron con la trampa cepa en pie tipo V, donde se logró capturar el mayor número de insectos adultos de Cosmopolites sordidus; la cual mostró diferencias significativas con los otros tres tratamientos. No se encontraron diferencias significativas entre los otros tres tratamientos. La trampa cepa en pie tipo $\mathrm{V}$ perdura y resiste mejor a las condiciones medioambientales, mientras que las otras trampas se descomponen aceleradamente, perdiendo su eficiencia.

PALABRAS CLAVES: Pseudotallo, trampa, picudo, Cosmopolites. 


\section{INTRODUCCIÓN}

El picudo negro del plátano (Cosmopolites sordidus, Germar 1824), es considerado como el insecto plaga más importante de las musáceas en Panamá y en el mundo. El daño principal causado por este insecto es en su fase larval; al alimentarse construyen galerías destruyendo de esta manera el tejido, ocasionando desbalances nutricionales en la planta, pudrición del área afectada, que conlleva algunas veces al acame de las plantas y por ende considerables pérdidas económicas. Hoy en día, el uso descontrolado de plaguicidas sintéticos en el control de plagas, ha ocasionado un desbalance ecológico y desarrollo de resistencia por parte de los organismos involucrados; así como también el encarecimiento de los costos de producción en la mayoría de los cultivos de la región; lo que conlleva a la búsqueda de otras alternativas para el manejo y control de plagas importantes, como parte de una estrategia de manejo integrado, reduciendo de igual manera el impacto ambiental que genera la actividad productiva. El plátano es un cultivo de mucha importancia a nivel de la provincia de Darién, representando la principal actividad económica de un número significativo de pequeños productores; por lo tanto, la generación de información científica orientada al manejo y control de problemas fitosanitarios en este cultivo, es de gran valor y ayuda para los productores. En esta dirección surge este proyecto de investigación enfocado en la evaluación la eficiencia de diferentes tipos de trampas de pseudotallo en la captura del picudo negro del plátano (Cosmopolites sordidus), en la provincia de Darién, República de Panamá. 


\section{PARTE EXPERIMENTAL}

\section{Área del ensayo:}

El trabajo se desarrolló en el Instituto Forestal Agropecuario de Darién (IFAD), el cual cuenta con parcelas de producción de plátano comercial y se encuentra ubicado en la comunidad de Canglón, Distrito de Pinogana, provincia de Darién.

\section{Diseño del ensayo:}

Se utilizaron parcelas en producción de la variedad Cuerno Blanco (AAB), susceptible al ataque del picudo del plátano. La unidad experimental correspondió a parcelas de $2,500 \mathrm{~m}^{2}$ cada una. Se establecieron cuatro tratamientos diferentes, correspondientes a tipos diferentes de trampas de pseudotallo, con cuatro repeticiones por tratamiento. Las trampas fueron evaluadas a los cuatro días después de instaladas, repitiendo el proceso durante tres periodos consecutivos, promediando el número de picudos capturados por tipo de trampa y repetición. La descripción de los tratamientos fue la siguiente:

T-1: Trampa de pseudotallo tipo sándwich cilíndrica o de disco.

T-2: Trampa de pseudotallo tipo sándwich - bisel.

T-3: Trampa de pseudotallo tipo cepa en pie tipo V.

T-4: Trampa de pseudotallo tipo de caída (Pitfall).

\section{Diseño experimental:}

El experimento se realizó bajo una distribución en bloques completamente al azar, con 4 tratamientos y 4 repeticiones por tratamiento; cada repetición estaba formada por 2 trampas, totalizando 8 trampas por tratamiento. La variable en evaluación correspondió al número de insectos capturados por tipo de trampa. Los datos obtenidos fueron transformados utilizando la fórmula $\mathrm{LOG}(\mathrm{X}+1)$ y se determinó el DMSt al 5\% de significancia. 


\section{RESULTADOS Y DISCUSIÓN}

El tratamiento correspondiente a la trampa de pseudotallo cepa en pie tipo V, permitió la captura del mayor número de insectos adultos. Los otros tratamientos no mostraron diferencias significativas entre ellos (Tabla 1).

Tabla 1: Resultados del DMSt al 5\% de significancia.

\section{HSD: $\quad 0.29$}

DATOS TRANSFORMADOS CON LA FÓRMULA LOG $(X+1)$

\begin{tabular}{|c|c|c|c|c|}
\cline { 2 - 5 } \multicolumn{1}{c|}{} & \multicolumn{4}{c|}{ TRATAMIENTOS } \\
\hline & Sandwish & Bisel & Cepa en Pie V & Pitfall \\
\hline 1 & 0.30 & 0.30 & 0.47 & 0.30 \\
\hline 2 & 0.30 & 0.30 & 0.95 & 0.30 \\
\hline 3 & 0.47 & 0.47 & 0.69 & 0.30 \\
\hline 4 & 0.30 & 0.30 & 0.47 & 0.47 \\
\hline
\end{tabular}

\section{Media}

$\begin{array}{lllll}\text { Aritmética } & 0.34 & 0.34 & 0.65 & 0.34\end{array}$

\begin{tabular}{|l|c|c|c|c|}
\hline & Sandwish & Bisel & $\begin{array}{c}\text { Cepa en } \\
\text { Pie V }\end{array}$ & Pitfall \\
\hline Sandwish & & 0 & -0.30 & 0 \\
\hline Bisel & & & -0.30 & 0 \\
\hline Cepa en Pie V & & & & 0.30 \\
\hline Pitfall & & & & \\
\hline
\end{tabular}


Estos resultados coinciden con los de trabajos de investigación realizados por Ajanel, (2003), Sandoval (2015), Medina \& Vallejo (2009), quienes evaluaron en diferentes localidades y países la efectividad y eficiencia de trampas de pseudotallo en la captura del picudo negro del plátano $C$. sordidus, obteniendo resultados similares a los nuestros, donde la trampa cepa en pie tipo $\mathrm{V}$ resultó ser más efectiva que el resto de trampas evaluadas. Durante las actividades de trampeo en campo, se pudo observar que la trampa de pseudotallo tipo de caída (pitfall), durante la temporada lluviosa pierde su

efectividad, ya que el recipiente donde permanecían los trozos de pseudotallo, se llena con el agua de lluvia, lo que pudo influir negativamente en la captura de los insectos adultos.

\section{CONCLUSIONES Y RECOMENDACIONES}

-La trampa de pseudotallo cepa en pie tipo V, es efectiva en la captura de insectos adultos de C. sordidus.

-El uso de trampas de pseudotallo cepa en pie tipo $\mathrm{V}$, representa una alternativa para el manejo y control de $C$. sordidus en el cultivo de plátano, como parte de una estrategia de manejo integrado de plagas en este importante rubro en la provincia de Darién.

-Es necesario replicar esta experiencia en otras áreas productoras de plátano en la provincia de Darién y el resto del país, buscando validar la efectividad y sostenibilidad en el tiempo de esta estrategia en el manejo de esta plaga. 


\section{SUMMARY}

A study was carried out to evaluate the efficiency of different types of pseudostem traps in the capture of adult Cosmopolites sordidus insects, in a commercial plantain plot in the province of Darién. The types of traps evaluated were; cylindrical sandwich, bevel type, V type foot strain and pitfall; determining the number of individuals caught per trap. The collected samples were analyzed in the Plant Protection Laboratory of the Faculty of Agricultural Sciences of the University of Panama. The experimental design was completely randomized blocks, with four treatments and four repetitions. The results obtained were subjected to the analysis of variance and the DMSt test, at a 5\% probability. The best results were obtained with the $\mathrm{V}$ type foot strain trap, where the highest number of adult Cosmopolites sordidus insects was captured; which showed significant differences with the other three treatments. No significant differences were found between the other three treatments. The $\mathrm{V}$ type foot strain trap, lasts and resists better to environmental conditions, while the other traps decompose rapidly, losing their efficiency.

Keywords. Pseudostem, trap, weevil, Cosmopolites.

\section{REFERENCIAS BIBLIOGRÁFICAS}

-Aguilera, L. 2002. Evaluación de seis tipos de trampas para el monitoreo y control del Picudo Negro (Cosmopolites sordidus) y Picudo Rayado (Metamasius hemipterus) en la plantación de plátano de Zamorano. Instituto Zamorano, Honduras. 35 p.

-Ajanel, O. 2003. Evaluación de tres tipos de trampas y cuatro frecuencias de recolección del picudo negro Cosmopolites sordidus (Germar 1824) en el cultivo de Banano Musa sapientum (Var. Grand nain) Tiquisate, Escuintla., Universidad de San Carlos de Guatemala. Guatemala. $61 \mathrm{p}$.

-Armendáriz, I.; Landázuri, P.; Ulloa, S. 2014. Buenas Prácticas para el Control del Picudo del Platano, Cosmopolites sordidus, en Ecuador. Ed. I.A., Quito, Ecuador. 21 p.

-Carballo, M. 2001. Opciones para el manejo del picudo negro del plátano. 
Manejo integrado de plagas CATIE, Hoja técnica No. 36. San José, Costa Rica. 4 p.

-Castrillón, C. 2003. Situación actual del picudo negro del banano (Cosmopolites sordidus. Germar) (Coleoptera: Curculionidae) en el mundo. In: Actas del Taller "Manejo convencional y alternativo de la sigatoka negra, nematodos y otras plagas asociadas al cultivo de musáceas", 2003. INIBAP, Guayaquil, Ecuador. p. 125-138.

-Govender P.; Viljonen A. 2002. Biología y manejo del picudo negro del banano, Cosmopolites sordidus, en África del Sur. Revista internacional sobre Banano y Plátano INFOMUSA. v.11, n.1., p10-18. Montpellier, Francia.

-Medina, C.; Vallejo, L. 2009. Métodos de muestreo para evaluar poblaciones de picudos del plátano (Coleoptera: Curculionidae, Dryophthorinae) en el departamento de Caldas-Colombia. Universidad de Caldas. Caldas, Colombia. 15p.

-Sandoval, M. 2015. Evaluación de tipos de trampas para la captura de Cosmopolites sordidus en el cultivo de banano. Universidad Rafael Landívar. Guatemala ,64 p. 